\title{
Eternal inflation and swampland conjectures
}

\author{
Hiroki Matsui ${ }^{1, *}$ and Fuminobu Takahashi ${ }^{1,2,3, \uparrow}$ \\ ${ }^{1}$ Department of Physics, Tohoku University, Sendai, Miyagi 980-8578, Japan \\ ${ }^{2}$ Kavli Institute for the Physics and Mathematics of the Universe (Kavli IPMU), UTIAS, WPI, \\ The University of Tokyo, Kashiwa, Chiba 277-8568, Japan \\ ${ }^{3}$ Center for Theoretical Physics, Massachusetts Institute of Technology, \\ Cambridge, Massachusetts 02139, USA
}

(Received 14 August 2018; published 30 January 2019)

\begin{abstract}
We study if eternal inflation is realized while satisfying the recently proposed string swampland criteria concerning the range of scalar field excursion, $|\Delta \phi|<\mathcal{D} \cdot M_{\mathrm{P}}$, and the potential gradient, $|\nabla V|>c \cdot V / M_{\mathrm{P}}$, where $\mathcal{D}$ and $c$ are constants of order unity and $M_{\mathrm{P}}$ is the reduced Planck mass. We find that only the eternal inflation of chaotic type is possible for $c \sim \mathcal{O}(0.01)$ and $1 / \mathcal{D} \sim \mathcal{O}(0.01)$ and that the Hubble parameter during the eternal inflation is parametrically close to the Planck scale and is in the range of $2 \pi c \lesssim H_{\text {inf }} / M_{\mathrm{P}}<1 / \sqrt{3}$.
\end{abstract}

DOI: $10.1103 /$ PhysRevD.99.023533

\section{INTRODUCTION}

The inflation is a quasi-de Sitter expansion of the Universe [1-5], which not only solves various initial condition problems of the standard big bang cosmology but also provides a natural explanation of the origin of density perturbations. Accumulating observational evidence supports that our Universe experienced inflation at an early stage of the evolution (see, e.g., Ref. [6]).

When the currently observable scales exited the horizon during inflation, the energy scale was much smaller than the Planck scale. Therefore, the inflation can be well described by a low-energy effective theory. This does not necessarily mean, however, that any inflation models realized in the language of the effective theory can be UV completed. In fact, it is widely believed that consistentlooking effective field theories could be in the swampland that cannot be consistently embedded in quantum gravity [7]. It is of utmost importance to have the correct criteria to identify the boundary between the landscape and the swampland.

Several conjectures on such criteria motivated by black hole physics [8] or string compactifications [9-11] have been proposed. Recently, it was proposed that an effective field theory that can be embedded consistently in quantum

\footnotetext{
*hiroki.matsui.c6@tohoku.ac.jp

†umi@tohoku.ac.jp
}

Published by the American Physical Society under the terms of the Creative Commons Attribution 4.0 International license. Further distribution of this work must maintain attribution to the author(s) and the published article's title, journal citation, and DOI. Funded by SCOAP ${ }^{3}$. gravity must satisfy the two conditions concerning the range of scalar field excursion and the potential gradient $[12,13] .{ }^{1}$ These conjectures are known to tightly constrain possible inflation theories, especially those based on a single-field slow-roll inflation regime. This lends support to more complicated inflation models or nonstandard history of the Universe such as chromonautral inflation [19], multifield inflation [20], or curvaton scenarios [21].

In this paper, we study if eternal inflation is realized while satisfying the above two swampland criteria. The eternal inflation [22-27] precedes the inflation epoch during which the currently observable scales exited the horizon, and it is considered to play an important role in populating various vacua (and therefore various theoretical possibilities) in the landscape. The eternal inflation can even explain a contrived fine-tuning of the parameters or setup that is necessary for the complicated life to emerge based on the anthropic argument. We will show in the later sections that only the eternal inflation of chaotic type is possible, and it requires that the numerical coefficients appearing in the swampland criteria are parametrically smaller or larger than unity.

\section{SWAMPLAND DISTANCE/DE SITTER CONJECTURES}

Here, we briefly summarize the two recently proposed swampland criteria. The first one is the swampland distance conjecture [12,28], which states that any effective field theories are valid only for a finite variation of the scalar

\footnotetext{
${ }^{1}$ We emphasize here that the conditions are still conjectures that have not been proven to be correct yet. In fact, there are many papers that criticize the conjectures [14-18].
} 
field, $\Delta \phi$. In the string compactification, the mass of an infinite tower of states (collectively denoted by $m$ ) becomes exponentially light in the field distance $\Delta \phi$,

$$
m^{2} \sim \exp \left(-\frac{\Delta \phi}{\mathcal{D} M_{\mathrm{P}}}\right)
$$

where $M_{\mathrm{P}} \simeq 2.4 \times 10^{18} \mathrm{GeV}$ is the reduced Planck mass and $\mathcal{D}$ is a numerical constant of order unity, but its precise value depends on details of the compactification. One can see that the large field deviation, $\Delta \phi \gtrsim \mathcal{D} M_{\mathrm{P}}$, would spoil the low-energy effective field theories, since a tower of the heavy states continuously becomes so light that they generally affect the low-energy dynamics. Thus, the following condition must be satisfied for the validity of the low-energy description,

$$
\frac{\left|\Delta m^{2}\right|}{m^{2}} \lesssim \mathcal{O}(1) \Rightarrow \Delta \phi \lesssim \mathcal{D} M_{\mathrm{P}},
$$

where the first inequality is such that heavy states should remain heavy during the scalar field excursion, $\Delta \phi$. For $\mathcal{D}=\mathcal{O}(1)$, the field variation $\Delta \phi$ cannot be much larger than the Planck mass. See, e.g., Refs. [28-32] for evidences supporting the conjecture.

The second one is the swampland de Sitter conjecture [13], which states that the low-energy effective potential, $V$, consistent with quantum gravity must satisfy

$$
|\nabla V|>c \frac{V}{M_{\mathrm{P}}}
$$

where $|\nabla V|$ is the norm of the gradient of $V$ on the scalar manifold and $c$ is a constant. The conjecture is based on recent discussions and criticisms about constructing de Sitter vacua in string theory (see, e.g., Ref. [33] for a recent review). The bound (3) forbids de Sitter vacuum solutions and also restricts possible slow-roll inflation scenarios. The validity of these conjectures and their cosmological implications have been recently discussed in Refs. [14-18,20,21,34-48].

The condition for inflation is formally written as

$$
\frac{\ddot{a}}{a}=H^{2}(1-\epsilon)>0 \Rightarrow \epsilon \equiv \frac{-\dot{H}}{H^{2}}<1,
$$

where $a(t)$ is the scale factor; the overdot denotes the derivative with respect to the cosmic time, $t$; and $H(t)=$ $\dot{a} / a$ is the Hubble parameter. The slow-roll condition requires $\epsilon<1$, while the conjecture (3) restricts the slow-roll parameter,

$$
\epsilon \simeq \frac{M_{\mathrm{P}}^{2}}{2}\left(\frac{\nabla V}{V}\right)^{2} \Rightarrow \epsilon^{1 / 2}>\frac{c}{\sqrt{2}}
$$

Thus, the slow-roll inflation requires $c<\sqrt{2}$.
In fact, the Planck measurements of the cosmic microwave background (CMB) [6] further restrict the slow-roll parameter as $\epsilon<0.0045$, which leads to $c<0.094$. Therefore, the condition (3) cannot be satisfied if $c$ is strictly equal to unity in a simple inflation model. Note that more complicated inflation models or nonstandard history of the Universe such as multifield inflation [20] or curvatonlike scenarios [21] can ameliorate the tension.

Lastly, we emphasize that the precise values of $c$ and $\mathcal{D}$ depend on the detailed string construction and they can be slightly larger or smaller than unity [43]. In the following sections, we study if eternal inflation can be realized while satisfying the above two swampland conjectures. In particular, we derive conditions on $c$ and $\mathcal{D}$, allowing possible deviations from their canonical values.

\section{ETERNAL INFLATION}

One of the most intriguing properties of the inflationary paradigm is that inflation can be eternal [22-26] (see also Ref. [27]). Once the eternal inflation begins, it never ends and continues to create an infinite number of the so-called bubble or pocket universes.

The eternal inflation occurs in a variety of setups, and they can be broadly classified into three categories: old inflation, hilltop inflation, and chaotic inflation. The old inflation is a classic example of eternal inflation. It takes place in a false vacuum, which collapses into one of the lower-energy vacua through tunneling and bubble formation, which is followed by slow-roll inflation. Inside such a bubble, the inflaton energy is transferred to a hot dense plasma after the slow-roll inflation ends. A collection of those bubble regions form pocket universes. The entire Universe, on the other hand, continues to inflate, populating an infinite number of the pocket universes. Our observable Universe is contained in one of the pocket universes.

If the fundamental theory has a large number of the false vacua (e.g., the string landscape [49]) with each having different values of the coupling constants and the cosmological constant [50-53], an apparently contrived finetuning of the parameters may be explained by anthropic arguments. The eternal inflation plays a crucial role in populating various vacua in the landscape.

The eternal hilltop or chaotic inflation takes place when quantum fluctuations of the inflaton dominate over its classical motion. In the case of eternal hilltop inflation $[22,23,54]$, it occurs in the vicinity of the local maximum of the potential where the classical motion vanishes, while in the case of eternal chaotic inflation [24-26], it occurs at large-field values for which the quantum fluctuation becomes significant. In either case, once the eternal inflation happens, it continues to inflate, which similarly helps to realize apparently fine-tuned parameters and/or initial conditions required for, e.g., the subsequent slow-roll inflation, curvaton scenarios, baryogenesis, etc. 
The swampland de Sitter conjecture (3) forbids de Sitter vacua or de Sitter extrema [13]. This immediately excludes the eternal old and hilltop inflation. ${ }^{2}$ In the following, therefore, we focus on the eternal inflation of chaotic type. A typical example of the chaotic inflation is characterized by potentials of the form $V(\phi) \propto \phi^{p}$ with large field excursion $\Delta \phi>M_{\mathrm{P}}$ [56]. ${ }^{3}$ The quantum fluctuations of the inflaton on the de Sitter spacetime are frozen after the horizon exit, and they can be considered as Brownian fluctuations or random walks of which the step size is equal to $H / 2 \pi$ with a time interval $H^{-1}$. The two-point correlation function $\left\langle\delta \phi^{2}\right\rangle$ is given by

$$
\left\langle\delta \phi^{2}\right\rangle=\frac{H^{3} t}{4 \pi^{2}}
$$

which grows with the cosmic time. Here, we approximate that the Hubble parameter is constant for simplicity, but we take into account the dependence of the Hubble parameter on the inflaton field in the next section.

To see how the eternal chaotic inflation takes place, let us consider a Hubble-size patch with a physical size $R \simeq H^{-1}$ where the inflation takes place. After the time interval $t$, this region exponentially expands to the size of $R \simeq H^{-1} \exp (H t)$, which contains $\mathcal{N}_{\text {patch }} \simeq \exp (3 H t)$ Hubble patches. The inflaton field takes a different value in each Hubble patch due to the accumulated quantum fluctuations. Let $P(\phi, t)$ denote the probability for the inflaton field to be equal to $\phi$ at the time $t$ in a Hubble patch. For an extreme case, let us estimate the probability for $\phi$ to increase and roll up the potential all the time during the time interval $t$. Such a probability $P(\phi, t)$ is approximately given by

$$
P(\phi, t) \sim\left(\frac{1}{2}\right)^{H t}=e^{-H t \ln 2}
$$

Thus, the probability exponentially decreases with the time, as expected. However, this does not necessarily mean that the number of such Hubble patches decreases with time, because the physical volume also increases as $\exp (3 \mathrm{Ht})$, which can compensate the exponentially small probability. Indeed, multiplying the volume factor, one obtains

\footnotetext{
${ }^{2}$ The (quasi-)de Sitter solution is subject to de Sitter instability from quantum backreaction. See, e.g., Ref. [55].

${ }^{3}$ Note that we consider the monomial inflaton potential during the eternal inflation, not during the last 50-60 $e$-foldings of inflation responsible for generating the observed density perturbations. Indeed, simple chaotic inflation models with quadratic or quartic potentials are already ruled out by the CMB observations [6], but this does not apply to the eternal inflation that had occurred well before the usual slow-roll inflation took place. See Refs. [57-59] for polynomial chaotic inflation and its supergravity realization, which give a better fit to the CMB data [60]. Also see Refs. [61-63].
}

$$
e^{H t \cdot(3-\ln 2)} \sim e^{2.3 H t} .
$$

This means that the inflaton field keeps increasing in some Hubble patches, the number of which is actually increasing due to the inflationary expansion.

In the above simplified discussion, we have neglected the classical motion of the inflaton and assumed that inflaton dynamics is dominated by the quantum jumps. We also did not consider its backreaction on the Hubble parameter. However, essentially the same thing can happen in a realistic case, as long as the quantum fluctuation is larger than the classical motion in a Hubble time. As a result, the inflaton can roll up the potential in some Hubble patches due to the fluctuations, and inflation continues to take place somewhere in the Universe. Remarkably, the eternal chaotic inflation does not require the potential minimum nor maximum, and in principle, it can satisfy the swampland conjectures. We will study this issue in more detail in the next section.

\section{ETERNAL CHAOTIC INFLATION AND SWAMPLAND}

In this section, we study if the eternal chaotic inflation can be realized while satisfying the swampland conjectures. As we have seen before, eternal inflation takes place if quantum fluctuations dominate over the classical variations of the inflaton field over the Hubble time [26,27]. Let us assume that inflation is driven by a single scalar field $\phi$. We define the slow-roll parameter as follows:

$$
\epsilon=\frac{M_{\mathrm{P}}^{2}}{2}\left(\frac{V^{\prime}(\phi)}{V(\phi)}\right)^{2}, \quad \eta=M_{\mathrm{P}}^{2} \frac{V^{\prime \prime}(\phi)}{V(\phi)} .
$$

If the above slow-roll parameters are much smaller than unity, the inflaton slowly rolls on the potential, and its dynamics is described by the equations

$$
3 H \dot{\phi} \simeq-V^{\prime}(\phi), \quad H^{2} \simeq \frac{V(\phi)}{3 M_{\mathrm{P}}^{2}},
$$

where the prime denotes the derivative with respect to $\phi$.

Neglecting the inflaton mass, the quantum fluctuations are approximately written as

$$
\langle\delta \phi\rangle_{\mathrm{q}} \approx \frac{H}{2 \pi} .
$$

On the other hand, the classical field variation over the Hubble time $H^{-1}$ is

$$
\langle\delta \phi\rangle_{\mathrm{c}}=H^{-1}|\dot{\phi}|
$$

The eternal inflation takes place if $\langle\delta \phi\rangle_{\mathrm{q}}$ is larger than $\langle\delta \phi\rangle_{\mathrm{c}}[26,27]$, 


$$
\frac{\langle\delta \phi\rangle_{\mathrm{q}}}{\langle\delta \phi\rangle_{\mathrm{c}}}=\frac{H^{2}}{2 \pi|\dot{\phi}|} \gtrsim 1
$$

Note that this implies that density perturbations of the modes that exited the horizon during eternal inflation have size of order unity, and the eternal inflation cannot be responsible for the density perturbations at the currently observable scales. We need another slow-roll inflation or other mechanism for generating the observed density perturbations.

Once the condition (13) is satisfied, eternal chaotic inflation takes place, which would populate many vacua in the landscape, if they exist. Using the slow-roll parameter $\epsilon$, one can rewrite the condition for eternal inflation as

$$
\frac{H}{M_{\mathrm{P}}} \gtrsim 2 \sqrt{2} \pi \epsilon^{1 / 2}
$$

By using the constraint (5) on $\epsilon$ from the swampland de Sitter conjecture, we arrive at

$$
\frac{H}{M_{\mathrm{P}}} \gtrsim 2 \pi c
$$

For the inflaton potential not to exceed the Planck energy, $V<M_{P}^{4}$, we also need $2 \sqrt{3} \pi c<1$. Thus, the eternal chaotic inflation is possible only if the Hubble parameter is parametrically close to the Planck scale unless $c$ is much smaller than unity, and quantum gravity effects may be significant. See, e.g., Refs. [1,55,64-67].

For example, if we assume a mild hierarchy, $H / M_{\mathrm{P}} \sim 0.1$, to suppress possible quantum gravity effects, the constant $c$ must be $c \lesssim 0.01$. which is even tighter than the CMB bound, $c<0.094$ [see the discussion below Eq. (5)]. This implies that, for the eternal inflation to be described by the classical treatment of the spacetime, one of the slow-roll parameters $\epsilon$ will be much smaller than the current upper bound.

Assuming the inflaton potential shape, one can estimate the inflaton field value where the eternal inflation is possible. In the case of the quartic potential, $V=\lambda \phi^{4}$, this is given by $\phi_{c} \simeq 3.5 \lambda^{-1 / 6} M_{\mathrm{P}}$. By combining (15), we obtain

$$
\mathcal{D} \gtrsim \frac{4}{\sqrt{3} c} .
$$

Thus, we need $c \lesssim \mathcal{O}(0.01)$ and $1 / \mathcal{D} \lesssim \mathcal{O}(0.01)$ for the eternal chaotic inflation to take place.

\section{DISCUSSION}

The quantum fluctuations on the expanding Universe can bring the inflaton field back to the initial state [25] or false vacuum [68]. Such a recycling process of the inflation makes the inflation eternal. Let us consider the possibility that the inflaton field at the end of the slow-roll inflation rolls up the potential by quantum fluctuations, following the argument of Ref. [51]. The probability density function $P(\phi)$ of finding the inflaton field of the value $\phi$ is given by a Gaussian distribution with the variance $\left\langle\delta \phi^{2}\right\rangle=H^{2} / 4 \pi^{2}$ for one Hubble time [69].

Now, we consider a situation in which at the end of the inflation the inflation field $\phi_{\text {end }}$ jumps up and becomes $\phi$ through a quantum jump in some Hubble patches. At the end of large-field inflation, the classical field variation during the Hubble time is of order the Planck mass, and so, the inflaton needs to jump upward by more than the Planck mass to overcome the classical motion. Let us denote the quantum jump by $C \cdot M_{\mathrm{P}}$ with $C \gtrsim 1$. Then, the probability for such a large quantum jump is given by

$$
P\left(\phi_{\mathrm{end}} \rightarrow \phi_{\mathrm{end}}+C M_{\mathrm{P}}\right) \approx \exp \left(\frac{-2 \pi^{2} C^{2} M_{\mathrm{P}}^{2}}{H_{\mathrm{end}}^{2}}\right)
$$

where we have used the Gaussian distribution with the variance $\left\langle\delta \phi^{2}\right\rangle=H^{2} / 4 \pi^{2}$. Suppose that, due to the previous inflationary expansion, there is a large number of the Hubble patches just before the inflaton experienced the large jump. The number of the Hubble patches that experience such a large jump is estimated as

$$
\mathcal{N}_{\text {patch }} \approx e^{3 N_{e}} P(\phi) \approx \exp \left(3 N_{e}-\frac{2 \pi^{2} C^{2} M_{\mathrm{P}}^{2}}{H_{\text {end }}^{2}}\right),
$$

where $N_{e}$ is the total $e$-folding number of the previous inflationary expansion. Thus, if we use $C \approx 1$, we obtain

$$
N_{e} \gtrsim \frac{\pi^{2}}{3} \frac{M_{\mathrm{P}}^{2}}{H_{\mathrm{end}}^{2}},
$$

to find more than one such Hubble patch. To go back to the inflaton field value at which the eternal inflation takes place, the total $e$-folding number must be even larger.

The required lower bound on the $e$-folding number is in tension with the swampland distance conjecture since it heavily restricts the duration of the inflation $[20,35,43]$. By using the swampland distance criterion and the Lyth bound [70], we connect the field variance $\Delta \phi$ and the total $e$-folding number $N_{e}$ as

$$
N_{e} \sqrt{2 \epsilon} \lesssim \frac{\Delta \phi}{M_{\mathrm{P}}} \Rightarrow 60 \lesssim N_{e} \lesssim \mathcal{D} / c
$$

where the lower bound of Eq. (19) comes from the cosmological observations to solve the horizon and flatness problems. Thus, the recycling process of the eternal inflation is also restricted by this bound. 


\section{CONCLUSIONS}

The eternal inflation is believed to play an important role in populating various vacua in the landscape if they exist. It also helps to realize apparently fine-tuned parameters or setups based on the anthropic arguments. In this paper, we have studied if the eternal inflation can be realized while satisfying the recently proposed swampland conjectures. We found that only the eternal inflation of chaotic type is possible. The Hubble parameter during the eternal inflation is parametrically close to the Planck scale, and it requires the numerical constants $c$ and $1 / \mathcal{D}$ appearing in the conjectures to be of $\mathcal{O}(0.01)$ or smaller. Even though the eternal inflation is not responsible for the observed density perturbations, the derived constraints on the numerical coefficients $c$ and $1 / \mathcal{D}$ have interesting implications for observables such as the tensor-to-scalar ratio as well as the dark energy equation of state through the swampland conjectures.

\section{ACKNOWLEDGMENTS}

F. T. thanks Prateek Agrawal for useful communications. F. T. is thankful for the hospitality of MIT Center for Theoretical Physics where this work was done. This work is partially supported by JSPS KAKENHI Grants No. JP15H05889 (F. T.), No. JP15K21733 (F. T.), No. JP17H02878 (F. T.), and No. JP17H02875 (H. M. and F. T.); by Leading Young Researcher Overseas Visit Program at Tohoku University (F. T.); and by World Premier International Research Center Initiative (WPI Initiative), MEXT, Japan (F. T.).
[1] A. A. Starobinsky, Phys. Lett. 91B, 99 (1980); 3, 130 (1987).

[2] A. H. Guth, Phys. Rev. D 23, 347 (1981).

[3] K. Sato, Mon. Not. R. Astron. Soc. 195, 467 (1981).

[4] A. D. Linde, Phys. Lett. 108B, 389 (1982).

[5] A. Albrecht and P. J. Steinhardt, Phys. Rev. Lett. 48, 1220 (1982).

[6] Y. Akrami et al. (Planck Collaboration), arXiv:1807.06211.

[7] C. Vafa, arXiv:hep-th/0509212.

[8] N. Arkani-Hamed, L. Motl, A. Nicolis, and C. Vafa, J. High Energy Phys. 06 (2007) 060.

[9] H. Ooguri and C. Vafa, Adv. Theor. Math. Phys. 21, 1787 (2017).

[10] B. Freivogel and M. Kleban, arXiv:1610.04564.

[11] T. D. Brennan, F. Carta, and C. Vafa, Proc. Sci. TASI2017 (2017) 015.

[12] H. Ooguri and C. Vafa, Nucl. Phys. B766, 21 (2007).

[13] G. Obied, H. Ooguri, L. Spodyneiko, and C. Vafa, arXiv: 1806.08362.

[14] M. Cicoli, S. De Alwis, A. Maharana, F. Muia, and F. Quevedo, Fortschr. Phys. 2018, 1800079 (2018).

[15] S. Kachru and S. P. Trivedi, arXiv:1808.08971.

[16] R. Kallosh, A. Linde, E. McDonough, and M. Scalisi, arXiv:1809.09018.

[17] R. Kallosh, A. Linde, E. McDonough, and M. Scalisi, Fortschr. Phys. 67, 1800068 (2018).

[18] Y. Akrami, R. Kallosh, A. Linde, and V. Vardanyan, Fortschr. Phys. 2018, 1800075 (2018).

[19] P. Agrawal, J. Fan, and M. Reece, J. High Energy Phys. 10 (2018) 193.

[20] A. Achucarro and G. A. Palma, arXiv:1807.04390.

[21] A. Kehagias and A. Riotto, arXiv:1807.05445.

[22] P. J. Steinhardt, in Very Early Universe, edited by G. W. Gibbons, S. W. Hawking, and S. T. C. Siklos (Cambridge University Press, Cambridge, 1983) pp. 251-266.

[23] A. Vilenkin, Phys. Rev. D 27, 2848 (1983).

[24] A. D. Linde, Mod. Phys. Lett. A 01, 81 (1986).
[25] A. D. Linde, Phys. Lett. B 175, 395 (1986).

[26] A. S. Goncharov, A. D. Linde, and V. F. Mukhanov, Int. J. Mod. Phys. A 02, 561 (1987).

[27] A. H. Guth, J. Phys. A 40, 6811 (2007).

[28] D. Klaewer and E. Palti, J. High Energy Phys. 01 (2017) 088 .

[29] R. Blumenhagen, I. Valenzuela, and F. Wolf, J. High Energy Phys. 07 (2017) 145.

[30] E. Palti, J. High Energy Phys. 08 (2017) 034.

[31] T. W. Grimm, E. Palti, and I. Valenzuela, J. High Energy Phys. 08 (2018) 143.

[32] B. Heidenreich, M. Reece, and T. Rudelius, Phys. Rev. Lett. 121, 051601 (2018).

[33] U. H. Danielsson and T. Van Riet, Int. J. Mod. Phys. D 27, 1830007 (2018).

[34] M. P. Hertzberg, S. Kachru, W. Taylor, and M. Tegmark, J. High Energy Phys. 12 (2007) 095.

[35] P. Agrawal, G. Obied, P. J. Steinhardt, and C. Vafa, Phys. Lett. B 784, 271 (2018).

[36] D. Andriot, Phys. Lett. B 785, 570 (2018).

[37] G. Dvali and C. Gomez, arXiv:1806.10877.

[38] A. Landete and G. Shiu, Phys. Rev. Lett. 121, 261301 (2018).

[39] S. Banerjee, U. Danielsson, G. Dibitetto, S. Giri, and M. Schillo, Phys. Rev. Lett. 121, 261301 (2018).

[40] L. Aalsma, M. Tournoy, J. P. van der Schaar, and B. Vercnocke, Phys. Rev. D 98, 086019 (2018).

[41] S. K. Garg and C. Krishnan, arXiv:1807.05193.

[42] J.-L. Lehners, J. Cosmol. Astropart. Phys. 11 (2018) 001.

[43] M. Dias, J. Frazer, A. Retolaza, and A. Westphal, arXiv: 1807.06579.

[44] F. Denef, A. Hebecker, and T. Wrase, Phys. Rev. D 98, 086004 (2018).

[45] E. O. Colgain, M. H. P. M. van Putten, and H. Yavartanoo, arXiv:1807.07451.

[46] C. Roupec and T. Wrase, arXiv:1807.09538.

[47] C. Roupec and T. Wrase, Fortschr. Phys. 67, 1800082 (2018). 
[48] D. Andriot, arXiv:1807.09698.

[49] L. Susskind, arXiv:hep-th/0302219.

[50] A. D. Linde and A. Mezhlumian, Phys. Lett. B 307, 25 (1993).

[51] A. D. Linde, D. A. Linde, and A. Mezhlumian, Phys. Rev. D 49, 1783 (1994).

[52] J. Garcia-Bellido, A. D. Linde, and D. A. Linde, Phys. Rev. D 50, 730 (1994).

[53] M. Tegmark, J. Cosmol. Astropart. Phys. 04 (2005) 001.

[54] G. Barenboim, W.-I. Park, and W. H. Kinney, J. Cosmol. Astropart. Phys. 05 (2016) 030.

[55] H. Matsui, arXiv:1806.10339.

[56] A. D. Linde, Phys. Lett. 129B, 177 (1983).

[57] K. Nakayama, F. Takahashi, and T. T. Yanagida, Phys. Lett. B 725, 111 (2013).

[58] K. Nakayama, F. Takahashi, and T. T. Yanagida, J. Cosmol. Astropart. Phys. 08 (2013) 038.

[59] K. Nakayama, F. Takahashi, and T. T. Yanagida, Phys. Lett. B 737, 151 (2014).
[60] C. Destri, H. J. de Vega, and N. G. Sanchez, Phys. Rev. D 77, 043509 (2008).

[61] A. D. Linde, Pis'ma Zh. Eksp. Teor. Fiz. 37, 606 (1983) [JETP Lett. 37, 724 (1983)].

[62] A. D. Linde, Phys. Lett. 132B, 317 (1983).

[63] R. Kallosh and A. D. Linde, J. Cosmol. Astropart. Phys. 04 (2007) 017.

[64] I. L. Buchbinder, S. D. Odintsov, and I. L. Shapiro, Effective Action in Quantum Gravity (IOP Publishing, Bristol, U.K., 1992).

[65] S. W. Hawking, T. Hertog, and H. S. Reall, Phys. Rev. D 63, 083504 (2001).

[66] I. L. Shapiro and J. Sola, Phys. Lett. B 530, 10 (2002).

[67] F. d. O. Salles and I. L. Shapiro, Phys. Rev. D 89, 084054 (2014); 90, 129903(E) (2014).

[68] J. Garriga and A. Vilenkin, Phys. Rev. D 57, 2230 (1998).

[69] A. D. Linde, Nucl. Phys. B372, 421 (1992).

[70] D. H. Lyth, Phys. Rev. Lett. 78, 1861 (1997). 